\title{
Deporte y emociones. Un estudio comparativo a partir del golf, el fútbol y la natación
}

\author{
Garriga Zucal, José \\ Instituto de Altos Estudios Sociales (IDAES) - Universidad Nacional de San Martín, \\ CONICET, Argentina \\ garrigajose@ hotmail.com \\ Hang, Julia \\ Centro de Investigaciones Sociohistóricas (CISH) - Facultad de Humanidades y Ciencias \\ de la Educación - Universidad Nacional de La Plata - CONICET, Argentina \\ julita.hang@gmail.com \\ Iuliano, Rodolfo \\ Centro Interdisciplinario de Metodología de las Ciencias Sociales (CIMeCS) - Facultad de \\ Humanidades y Ciencias de la Educación - Universidad Nacional de La Plata, Argentina \\ rodolfoiuliano@gmail.com
}

Cita sugerida: Garriga Zucal, J., Hang, J. y luliano, R.(2018). Deporte y emociones. Un estudio comparativo a partir del golf, el fútbol y la natación. Cuestiones de Sociología, 18, e053. https://doi.org/10.24215/23468904e053 


\section{Deporte y emociones. Un estudio comparativo a partir del golf, el fútbol y la natación}

Sports and emotions. A comparative study from golf, football and swimming

José Garriga Zucal

Instituto de Altos Estudios Sociales (IDAES) - Universidad

Nacional de San Martin, CONICET, Argentina

garrigajose@hotmail.com

Julia Hang

Centro de Investigaciones Sociobistóricas (CISH) - Facultad

de Humanidades y Ciencias de la Educación - Universidad

Nacional de La Plata - CONICET, Argentina

julita.hang@gmail.com

Rodolfo Iuliano

Centro Interdisciplinario de Metodología de las Ciencias

Sociales (CIMeCS) - Facultad de Humanidades y Ciencias

de la Educación - Universidad Nacional de La Plata,

Argentina

rodolfoiuliano@gmail.com

\section{Resumen:}

Este artículo presenta una reflexión sobre la relación entre deporte y emociones. Analizando comparativamente el mundo del golf, el de los nadadores master y el de los espectadores del fútbol en la Argentina se busca comprender los modos en que el deporte permite el control de las emociones por un lado, y por otro, habilita la emergencia de las mismas. Nos interesa ahondar en lógicas diferentes para desnaturalizar miradas esencialistas, pensando a las emociones en su relación con la "locura", la violencia y la técnica deportiva.

Palabras clave: Deporte, Emociones, Locura, Violencia, Técnica Deportiva.

\section{Abstract:}

This article thinks about the relationship between sport and emotions. Analyzing comparatively the worlds of golf, master swimmers and football fans in Argentina, we seek to understand the ways in which sport allows the control of emotions on the one hand, and on the other, affords their emergence. We are interested in deepen into different logics to denaturalize essentialist views, thinking of emotions in their relationship with "madness", violence and sports technique.

KEYwORDs: Sports, Emotions, Madness, Violence, Sports techinques .

\section{INTRODUCCIÓN}

En este artículo nos proponemos reflexionar, en clave comparativa, sobre la relación entre deporte y emociones. El estudio sobre estos vínculos tiene larga data y encuentra su punto de inicio en los trabajos de Norbert Elias (Elias y Dunning, 1995). Si analizamos el mundo del golf, el de los nadadores master y el de los espectadores del fútbol en la Argentina, proponemos reflexionar sobre los modos en que el deporte permite el control de las emociones, por un lado, y por otro habilita la emergencia de las mismas. Nos interesa ahondar en lógicas diferentes para desnaturalizar miradas esencialistas, pensando a las emociones en su relación con la "locura", la violencia y la técnica deportiva. 


\section{GOBIERNO EMOCIONAL, CAJA DE RESONANCIA Y HABILITACIÓN TÉCNICA EN EL GOLF}

La práctica del golf suele ser representada en una aparente paradoja como irracional y al mismo tiempo calculatoria. Las personas que juegan al golf aparecen representadas con frecuencia o bien como "locos" que pierden su tiempo en una actividad "sin sentido", o bien como "interesados" que lo explotan para fines siempre sospechosos. Cuando tenemos oportunidad de escuchar a las personas que juegan al golf, ellas relatan su afición estableciendo vinculaciones entre el juego y las emociones. Algunos de los sentidos que asume esa vinculación remiten a nociones sobre lo placentero, lo lúdico, lo adictivo (Iuliano, 2012). Sin embargo, no ha sido suficientemente explorado el modo en que la práctica del golf permite a los jugadores movilizar determinados estados emocionales y experimentar emociones durante la práctica.

Una tarde de mayo de 2016 Leopoldo había ido al club a "tirar un canasto de pelotas" en la cancha de práctica. Ese domingo, el amplio estacionamiento del club estaba colmado de autos y había mucha gente jugando un torneo. Predominaba el silencio, sólo interrumpido por el sonido suave de los neumáticos rodando contra el ripio de la playa de estacionamiento, o por el sonido metálico del impacto del palo sobre las pelotas de golf en la cancha de práctica. Luego de tirar dos canastos de pelotas durante casi una hora Leopoldo dijo "listo, necesitaba tirar unos baldes. Hace un rato discutimos con mi mujer y venía re caliente. Ahora me voy más tranquilo”. Se subió a su auto y se fue del club.

Otra tarde, mientras uno de los jugadores a los que estaba acompañando en la cancha de golf se encontraba afirmado en su stand ${ }^{1}$ armando el back swing ${ }^{2}$ para golpear la pelota, escuchamos a la distancia unos sonidos estridentes y unos gritos. Los tres nos sobresaltamos y nos detuvimos para observar cómo, a unos treinta metros y en otro fairway ${ }^{3}$, un hombre de unos cincuenta años alzaba su palo con las dos manos estirándose en posición vertical, y lo bajaba repetidas veces con violencia en un movimiento similar al de un leñador, golpeándolo contra una bolsa de palos (que asumimos era suya), profiriendo insultos y otras palabras que no alcanzábamos a discernir.

Al conversar sobre lo sucedido con otro jugador, me dijo

Yo me pregunto por qué razón te calentás tanto con el golf. Debe ser porque le tenés que pegar a una pelota que está quieta y no podés. Tiene mucha adrenalina. Debe ser por eso que los deportistas que están sometidos a mucha presión como los futbolistas cuando se retiran juegan al golf. Yo a veces tiro el palo. No me da para romperlos porque sé lo que salen, pero a veces lo tiro a la bolsa. Un día te salió todo y vas al otro día y no le pegás. Es todo psicológico, yo cuando tengo algún problema en el trabajo ya sé que me va a ir mal en el golf. Y es así, no te sale nada. Y cuando estás bien en el trabajo y con la familia, vas y jugás bárbaro.

La práctica del golf opera sobre el estado emocional del sujeto. La secuencia repetitiva de golpes a una pelota con un palo de golf se constituye en un instrumento del gobierno emocional; el sujeto planifica y ejecuta una intervención regulatoria de su estado anímico capaz de aplacar un malestar surgido de una pelea doméstica. La regulación de las emociones no es exclusivamente un efecto figuracional del proceso civilizatorio (Elias, 1994), sino un ejercicio activo de los sujetos, quienes conocen los discursos contemporáneos sobre la emocionalidad que interviene sobre sus estados de ánimo haciéndolos actuar (Hennion, 2010, 2017; Semán y Boix, 2017) y los moviliza de acuerdo. Al mismo tiempo, el juego puede detonar en el jugador manifestaciones de violencia como insultar y gritar; o golpear, tirar y romper el equipo. Es interesante observar cómo los propios actores enuncian una teoría sobre la vinculación entre la práctica deportiva y las emociones, que comprende diferentes elementos explicativos: por un lado, la distancia entre la aparente simplicidad del juego y la dificultad para dominar de forma regular la técnica deportiva habilitaría la "calentura" y dispondría un medio hormonal adrenalínico durante el cual transcurre la práctica; por otro lado, se establece una continuidad psicológica entre los diferentes dominios (familiares, laborales, deportivos) de la vida subjetiva, donde los estados emocionales van resonando como un encadenamiento de ecos. 
Si atendemos a la experiencia y al relato de los practicantes, es posible observar el modo en que se entraman y producen algunas emocionalidades contemporáneas. En primer lugar, vemos que esta práctica deportiva, que ocurre en un entorno material caracterizado por el silencio y el relativo aislamiento urbano, es concebida por los sujetos como una técnica de producción emocional capaz de intervenir y modificar un estado anímico precedente. En segundo lugar, y en dirección inversa, el fenómeno deportivo es entendido como una caja de resonancia, como una instancia expresiva donde se manifiestan los estados emocionales que tienen lugar en la subjetividad del deportista. Finalmente, la singularidad técnica del golf que lo deshomologiza respecto de otras disciplinas deportivas, dispone al aficionado en una vinculación emocional apasionada y desbordada al mismo tiempo.

\section{EMOCIONES Y VIOLENCIA(S) EN EL FÚTBOL}

Cuando se tratan las acciones de las denominadas "barras" las emociones son una muestra de discursos contradictorios. Las violencias de las "barras" pueden ser presentadas y representadas como desborde emocional o, por el contrario, como estrategia racional de un cálculo de la acción. De la ausencia total de regulación al control total de las pasiones, así son representados los "barras"(Garriga Zucal, 2007). Durante la investigación descubrimos un juego más interesante y complejo: una regulación situacional de diferentes emociones. Las violencias no son ejemplo de incapacidad de autocontrol sino el resultado de relaciones que legitiman prácticas de forma diferencial según las interacciones.

Los miembros de la "barra" desean ser definidos como aguantadores. El aguante tiene que ver, para ellos, con piñas, patadas y pedradas, con soportar los gases lacrimógenos y otros efectos de la represión policial, con cuerpos luchando y resistiendo el dolor. Pelear, afrontar con valentía y coraje una lucha corporal, es prueba de la posesión del aguante. El honor grupal e individual se gana o pierde en acciones violentas. Sin embargo, no son todas las acciones violentas son válidas. Existe un conjunto de prácticas legítimas y otras ilegítimas. Por ejemplo, está mal considerado el enfrentamiento físico con espectadores que nos sean parte de las "barras". Es decir: está bien pelearse pero no con todo el mundo. Notamos, entonces, que las acciones violentas están altamente reguladas y contenidas/moldeadas por los valores grupalmente aceptados.

Una cuestión más ilumina la regulación de las emociones. Los actores sociales que cometen hechos violentos en el mundo del fútbol lo hacen como parte de un entramado social complejo que legitima esas acciones, en esos contextos. Estos actores, en otros contextos, actúan de otras formas, es decir, no es la violencia una particularidad natural sino una acción -legítima y válida- que, usada como recurso social, les permite ubicarse en un determinado espacio social. A modo de ejemplo, recordemos el caso de un miembro de la "barra" que los sábados peleaba en los estadios y los domingos formaba parte de una agrupación católica como los boy scout. Dos contextos relacionales disímiles regulaban la acción de forma diferente.

Hemos brevemente presentado como la participación en la "barra" tolera la exteriorización de ciertas formas violentas inhibidas en otras tramas relacionales. Al mismo tiempo y eficazmente regula otras emociones. Evidencia, así, formas de autocontrol compartidas con el resto de los actores sociales con los que se vinculan e interactúan.

El aguante es un dispositivo de regulación de las emociones. Advertimos la existencia de grupos que elaboran esquemas de percepción del mundo -contradictorios y asistemáticos- basados en la aceptación de algunas formas de violencia. Las violencias legítimas no son la desregulación de las emociones, ni mucho menos. Las prácticas violentas son acciones que los grupos usan para comunicar variados aspectos de su cosmovisión, desde la masculinidad a la idealización de un modelo de cuerpo, desde la entereza de espíritu a la resistencia al dolor como valor ontológico. 


\section{PASIÓN, LOCURA Y PLACER SENSIBLE EN TORNO A LA EXPERIENCIA DE LA NATACIÓN MASTER}

La práctica de la natación master ${ }^{4}$ en personas adultas representa un espacio caracterizado, desde el punto de vista de sus practicantes, por la armoniosa combinación entre la dimensión "social” y la "deportiva”. En un trabajo realizado con nadadores master de un equipo de la ciudad de La Plata dimos cuenta del modo en que personas adultas ponen a jugar, en torno a la natación, representaciones sobre la salud, la amistad y el placer que contribuyen a configurar un universo moral particular (Hang, 2011).

Allí conocimos a Lidia, una bioquímica que hoy tiene 64 años, y que hace ya veinte años que participa del equipo de natación master, en el que comenzó por una recomendación médica para tratar un problema óseo. Incentivada por su profesora del nivel inicial, se fue "enganchando" hasta animarse a competir. Si bien en un principio "la parte competitiva" no le interesó, le atrajo "la parte social" ya que se define como una persona "sociable y muy charlatana" a la que le encanta conocer gente nueva. Para Lidia, la práctica de la natación master se convirtió en una pasión. No sólo por los vínculos generados en la práctica que desbordan ese espacio y pasan a ser parte del resto de sus actividades de tiempo libre, sino también por el goce que le genera la adquisición y puesta en práctica de una destreza corporal. Ella es múltiple campeona nacional y sudamericana en el estilo mariposa en las distancias de 50, 100 y 200 metros, estilo que le "fascina". Así, explica que "no siento en alguna actividad deportiva tanto placer como es el deslizarme en mariposa. Me fascina. Sobre todo cuando estoy entrenada, y no me canso. El ir, venir, me enloquece.” Así, el relato de Lidia nos permite encontrar el placer de la actividad física en un proceso por el cual hay que pasar (cuando no está entrenada) hasta llegar a un momento en el que con un entrenamiento adecuado se lograría el goce en la actividad ("sobre todo cuando estoy entrenada y no me canso"). Relatos como el de Lidia, donde se destacan los aspectos del placer y el goce, nos obligan a tomar en serio la materialidad, la productividad específica de este tipo de prácticas. Y este placer experimentado, para ser explicado con palabras, requiere de una analogía, en este caso, de la comparación con el "volar". Lidia refiere que "ese deslizar de mariposa que me fascina, es una cosa hermosísima. Debe ser como el volar, como el vuelo de un pájaro, ese aletear, bueno, yo lo comparo de cierta manera con eso y me gusta."

Mientras que durante mucho tiempo, las prácticas deportivas han sido entendidas desde una perspectiva instrumental, desde una funcionalidad en relación con la reproducción de las relaciones de clase, de las prácticas de consumo y la colonización capitalista del tiempo libre como zonas en las cuales se reproduce la fuerza de trabajo del capitalismo contemporáneo (Foucault, 2008; Brohm, 1993), aquí, por el contrario, emerge el lugar de la agencia de los sujetos, de su capacidad para experimentar sensaciones placenteras en este tipo de actividades, aún en algo que parecería rutinario como "el ir y venir". Y en este testimonio en particular, se pone en evidencia aún más el modo en que esa materialidad asume una forma corporal, una forma sensible, donde el placer aparece asociado a una actividad corporal característica de los nadadores cuando adquieren y mejoran un "estilo" que es el "deslizar", es decir, el desplazarse por el agua con cierta naturalidad, con cierta fluidez, percibiendo cierta armonía entre el cuerpo y el agua. Esta dimensión sensible de la experiencia en torno a esta práctica deportiva aparece referida también por analogía, a otra actividad físico/corporal como es el vuelo de un pájaro.

Desde hace un par de años Lidia participa de "competencias en aguas frías". Junto con un grupo de nadadores de varios lugares del país, realizan travesías en lagos del sur del país, ríos y mares, con la particularidad de no utilizar el traje de neoprene que los resguardaría del frío. Si antes le "enloquecía" nadar mariposa, esta locura debe ser ahora resignificada en las aguas frías. "Nos dicen que estamos locos por nadar en aguas heladas, pero sabemos lo que hacemos. Estamos asesorados por médicos y entrenadores" me aclara Lidia. Lo que para "los otros" aparecería como irracional, para Lidia son "mitos y consejos de los que no se animan, que hay que desarmar rompiendo el hielo”. Esta imagen, que refiere a una experiencia metafórica pero también sensible (la frase fue acompañada de una fotografía en la cual Lidia aparece nadando en el canal de Beagle rodeada de trozos de hielo flotando) pone de manifiesto la singularidad de la práctica, a la que a 
la sensación de volar se le suma ahora el contacto corporal con el agua helada, y al mismo tiempo, la belleza del paisaje. "Es maravilloso nadar con éste paisaje, es un desafío grosso por la temperatura del agua, pero cuando logras controlar la respiración, y no ponerte loca te das cuenta que es posible hacerlo” me comentó orgullosa sobre su travesía en las aguas del "fin del mundo". Y en esta actividad particular se puede observar como el placer aparece regulado y orientado, en este caso, por médicos y entrenadores que la asesoran para que pueda "controlar la respiración y no volverse loca". Porque tanto para Lidia como para los nadadores de aguas frías, la clave para ser exitoso en esta disciplina pasa por "el control de la mente, pensar que podemos, pero mantenernos tranquilos". El placer experimentado en las aguas frías parece ser aprendido en el tránsito por una carrera (Becker, 2009), en la cual el control sobre las emociones (sobre el cuerpo y la mente) tiene un lugar central.

\section{CONCLUSIONES}

Retomemos los tres bloques para agilizar la comparación. A pesar de su relativa popularización - a escala global y, también, en la Argentina- el golf sigue siendo pensado como un deporte de elite. La mirada miserabilista del sentido común tiende a representar a las prácticas deportivas de las categorías superiores como racionales, espacio de regulación de las emociones. Por el contrario, las prácticas de los sectores populares son representadas como emocionales o violentas. Al abordar las dimensiones de gobierno emocional, caja de resonancia y habilitación técnica que se anudan en el dispositivo del golf contribuimos a avanzar en la problematización empírica de las representaciones sociales y eruditas que proyecta en las prácticas deportivas "exclusivas" de las categorías superiores un territorio pleno de racionalidad y cálculo, expurgado de emocionalidad, e incluso, de toda violencia. La comparación con los miembros de la "barra" del fútbol da cuenta tanto del carácter socialmente construido de la dicotomía civilización y violencia, como del carácter lógico, racional y civilizatorio que estructura ciertas prácticas violentas entre los sectores medios y populares. Además, en la comparación con los golfistas la representación del control de emociones asociado a la "locura" entre los nadadores master permite evaluar y reflexionar sobre la racionalidad de las prácticas deportivas. En los tres casos se observa un control sobre ciertas emociones - áreas reguladas- que permiten complejizar la reflexión sobre deporte y emociones tirando por tierra las asociaciones simplistas entre clase y autocontrol. Y deja al descubierto una búsqueda -diferente según grupos y contextos- de experiencias emotivas que pueden ser interpretadas, sin esfuerzo, como irracionales por cualquier observador externo.

\section{REFERENCIAS}

Becker, H. (2009). Outsiders. Hacia una Sociología de la Desviación [1963]. Buenos Aires: Siglo XXI Editores.

Brohm, J.M. (1993). 20 tesis sobre el deporte. En AA. VV, Materiales de sociología del deporte (47-55). Madrid: La Piqueta.

Elias, N. (1994). El proceso civilizatorio. Méxcio: FCE [1939].

Elias, N., y Dunning, E. (1995). Deporte y ocio en el proceso de la civilización. México: FCE [1986]

Foucault, M. (2008). Vigilar y castigar: nacimiento de la prisión. Buenos Aires: Siglo XXI.

Garriga Zucal, J. A. (2007). Haciendo amigos a las piñas. Violencia y redes sociales de una hinchada de fútbol. Buenos Aires: Prometeo.

Hang, J. (2011): 'Lo que pasa en los master queda en los master': Deporte, sociabilidad y clasificaciones morales entre nadadores master del Club Universitario de La Plata (Tesis de grado), Universidad Nacional de La Plata, Facultad de Humanidades y Ciencias de la Educación para optar al grado de Licenciada en Sociología. Disponible en: http://www.memoria.fahce.unlp.edu.ar/tesis/te.718/te.718.pdf 
Hennion, A. (2010). Gustos musicales: de una sociología de la mediación a una pragmática del gusto. Comunicar, 17(34), 25-33. Disponible en: https://www.revistacomunicar.com/index.php?contenido=detalles\&numero=3 4\&articulo=34-2010-04

Hennion, A. (2017). De una sociología de la mediación a una pragmática de las vinculaciones. Retrospectiva de un recorrido sociológico dentro del CSI. Cuestiones de Sociologia, 16 (185-212) Disponible en: http://www.cuesti onessociologia.fahce.unlp.edu.ar/article/view/CSe032/8524

Iuliano, R. (2012). Mirá el día de golf que me hiciste perder. Emociones y aficiones en torno a la práctica del golf. En Actas de las II Jornadas de Antropología, PPGAS, IFCH-UNICAMP, Campinas, Brasil, Disponible en http:/ /historiapolitica.com/datos/biblioteca/estudiosdeportes_iuliano.pdf

Semán, P., y Boix, O. (2017), Mediaciones y Pragmatismo. Cuestiones De Sociología, 16 (175-184) Disponible en: htt p://www.cuestionessociologia.fahce.unlp.edu.ar/article/view/CSe031/8529

\section{Notas}

1 Posición desde la cual se ejecuta el golpe.

2 Movimiento pendular de los brazos que se encuentran sujetando el palo de golf para tomar impulso durante la ejecución del golpe.

3 Zona de la cancha de golf libre de pastizales y arboledas, dispuesta para que se deslicen las pelotas luego de los golpes largos y para que los jugadores se desplacen durante el juego.

4 La natación master es practicada por personas adultas, mayores de 25 años, que están dispuestas a competir en campeonatos nacionales e internacionales, cuyas categorías se dividen por sexo y edad. 